Background: Quantitative MRI provides important information about tissue properties in brain and how they maybe affected by degenerative disorders. Alzheimer's disease (AD) can be characterized by a specific pattern of atrophy, and a faster rate of tissue changes than controls. However, the precise spatiotemporal patterns of quantitative MRI in AD are unknown. We aimed to investigate neuroimaging correlates of $\mathrm{AD}$ using serial quantitative MRI. Methods: In our study, subjects with $\mathrm{AD}(\mathrm{N}=21)$ and similar-aged healthy controls $(\mathrm{N}=32)$ underwent two serial brain MRI scans at baseline and 12 months. Tissue characteristics were captured using two quantitative MRI parameters: longitudinal relaxation time (qT1) and transverse relaxation time (qT2). Results: The AD and controls groups were statistically compared using a voxel based quantification method. At baseline, subjects with $\mathrm{AD}$ showed a significant reduction of qT1 and qT2 compared to controls in bilateral temporal and parietal lobes, hippocampus, and basal ganglia (c.f. Fig 1A). This pattern was also observed at follow-up. Longitudinally, in $\mathrm{AD}$ we found a significant increase rather than further reduction of qT1 and qT2 from the baseline in bilateral hippocampus, thalamus and right caudate nucleus (c.f. Fig 1B\&C). In addition, the longitudinal change of qT1 in left hippocampus was negatively correlated with cognitive decline in $\mathrm{AD}$ over the 1year period, and the general disease severity significantly predicted the amount of increase of qT1 in bilateral hippocampus over 12 months. The longitudinal change of qT2 in left parahippocampus correlated with change in neuropsychiatric features over time. Conclusions: Quantitative MRI parameters were reduced in AD cross-sectionally, but increased over time, showing distinct spatiotemporal patterns from the atrophy in AD. We also showed the clinical relevance of quantitative MRI parameters, indicating their potential utility as new imaging markers in AD.
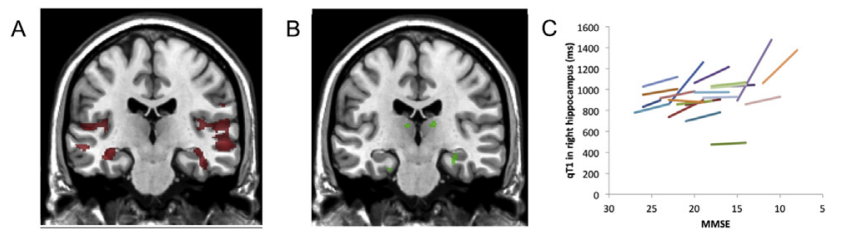

\section{IC-P-076 LESS DAILY COMPUTER USE IS RELATED TO SMALLER HIPPOCAMPAL VOLUMES IN DEMENTIA-FREE ELDERLY}

Lisa C. Silbert ${ }^{1}$, David Lahna ${ }^{1}$, Hiroko H. Dodge ${ }^{1,2}$,

Nutta-on Promjunyakul ${ }^{1}$, Nora Mattek ${ }^{1}$, Deniz Erten-Lyons ${ }^{1}$, Daniel Austin ${ }^{1}$, Jeffrey Kaye ${ }^{1},{ }^{1}$ Oregon Health \& Science University, Portland, OR, USA; ${ }^{2}$ University of Michigan, Ann Arbor, MI, USA. Contacte-mail: silbertl@ohsu.edu

Background: Unobtrusive continuous in-home monitoring may be a more sensitive means of detecting early functional changes associated with conversion to mild cognitive impairment and dementia in older individuals than traditional testing methods acquired sporadically over time. Methods: Twenty-nine dementia-free elderly volunteers (mean age 87.4, MMSE 28.2) enrolled in the Intelligent Systems for Assessing Aging Change study underwent 3T brain MRI. FreeSurfer was used to determine gray matter (GM) and hippocampal volumes. White matter hyperintensities (WMH) were derived using customized software. In-home computer use (CPU) was calculated using daily mouse movement detection and averaged over a one month period surrounding the time of their MRI visit. A multivariate logistic regression was performed with outcome of either high $(>25 \mathrm{~min} /$ day $)$ or low $(<25 \mathrm{~min} /$ day $)$ average computer use. A voxel based morphometry analysis was also performed to identify relationships between CPU and regional GM density. T1 images were brain extracted and then linearly aligned to the MNI-152 template and averaged to create a study specific template. All T1 images were then non-linearly aligned to this template while the degree of expansion or contraction was calculated. This transform was then applied to segmented GM tissue masks which were modulated by the Jacobian and then used for permutation testing in a model which also accounted for age and gender. Results: In the univariate analysis, less CPU was associated with smaller hippocampal volumes, but not with either total GM or WMH volumes. In a multivariate regression adjusted for age, gender, and intracranial volume, less CPU was associated with smaller hippocampal volume $(\mathrm{p}=0.027)$. Voxel-wise analysis demonstrated that greater CPU was associated with increased GM density in the: right hippocampus, left hippocampus, left lingual gyrus, right inferior temporal gyrus, left superior temporal gyrus and right cingulate gyrus. Conclusions: Daily computer use is related to brain regions linked to memory function and the processing of visual and spatial stimuli, areas previously shown to be associated with conversion to Alzheimer's dementia. Findings support the use of continuous in-home monitoring as a sensitive method to detect meaningful changes in older individuals at risk for dementia.

Table 1

Regions in which VBM identified significantly reduced GM density in comparison with less daily computer use.

\begin{tabular}{|c|c|c|c|c|c|c|}
\hline \multirow[b]{2}{*}{ Talairach Region } & \multicolumn{4}{|c|}{ Coordinates } & \multicolumn{2}{|c|}{ Cluster Size } \\
\hline & Side & $\mathrm{X}$ & $\mathrm{Y}$ & Z & Voxels & Volume $\left(\mathrm{mm}^{3}\right)$ \\
\hline Hippocampus & Rt & -28.4 & 9.3 & -21 & 1044 & 8352 \\
\hline Hippocampus & $\mathrm{Lt}$ & 25.7 & 9.7 & -18.7 & 770 & 6160 \\
\hline Lingual Gyrus & $\mathrm{Lt}$ & 10.3 & 74.8 & 4.7 & 99 & 792 \\
\hline Inferior Temporal Gyrus & Rt & -63.5 & 28.1 & -19.3 & 94 & 752 \\
\hline Superior Temporal Gyrus & $\mathrm{Lt}$ & 46.9 & -9.9 & -20.1 & 81 & 648 \\
\hline Cingulate Gyrus & Rt & -1 & -14 & 38.8 & 53 & 424 \\
\hline Middle Temporal Gyrus & $\mathrm{Lt}$ & 43.1 & -5.7 & -35.8 & 25 & 200 \\
\hline
\end{tabular}

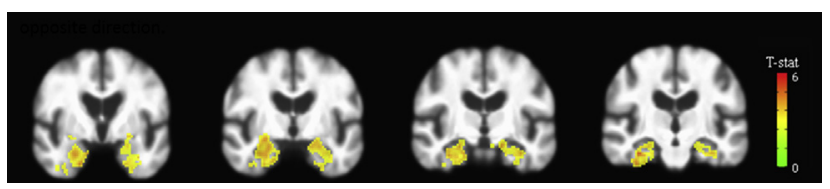

Figure 1. VBM analysis. Colored voxels indicate regions of decreased GM density significantly associated with less daily computer use $(\mathrm{p}<0.05)$.

\section{IC-P-077 HIPPOCAMPAL MRI TEXTURE IS RELATED TO HIPPOCAMPAL GLUCOSE METABOLISM}

Lauge Sørensen ${ }^{1,2}$, Christian Igel ${ }^{1}$, Mads Nielsen ${ }^{1,2},{ }^{1}$ University of Copenhagen, Copenhagen, Denmark; ${ }^{2}$ Biomediq A/S, Copenhagen, Denmark.Contacte-mail:madsn@biomediq.com

Background: We previously saw that hippocampal magnetic resonance imaging (MRI) texture provided-volume independent information in Alzheimer's disease (AD) diagnosis (AAIC 2012, 